\title{
Theoretical Foundations of Mobile Flexible Networks
}

\author{
Mérouane Debbah \\ Alcatel-Lucent Chair on Flexible Radio, SUPELEC, Gif sur Yvette Cedex, France \\ Correspondence: merouane.debbah@supelec.fr \\ Manuscript communication: received 29 March 2010, accepted 16 December 2010
}

\begin{abstract}
The general framework of Mobile Flexible Networks (MFN) is to design dense self-organizing, self-healing and self-energy harvesting secure networks where terminals and base stations interact and self-adapt in an intelligent manner without the need of a central controller or with the right amount of regulation to let the agents in the network exploit fully the degrees of freedom. The design depends mainly on the mobility pattern as in highly mobile environments, intelligence at the terminal reduces the cost of signaling whereas for fixedling whereas for fixed (non-mobile) networks, all the intelligence can be put on the network side. One of the big challenges is to find how to optimally split the intelligence between cognitive terminals and cognitive networks. In this paper, we discuss the challenges ahead and provide some research directions to develop the theoretical foundations of these networks.
\end{abstract}

Keywords- Flexible networks, self-organization, cognitive networks, wireless, MIMO.

Part of this work was presented at the 2008 IEEE International Conference on Advanced Technologies for Communications, Hanoi, Vietnam.

\section{INTRODUCTION}

Imagine a highway which dynamically switches the number of dedicated downstream and upstream lanes according to the observed car traffic. The highway would go from two to three lanes if the traffic is dense. It would also signal to the different car drivers, depending on their needs, changes in their directions in order to ease the traffic process. It would coordinate for example with other highways the traffic lights for scheduling the cars accordingly. It could also transform the road material (from smooth to harsh), to force the users to reduce their speed depending on the weather conditions for security reasons. Finally, in the case of electrical cars, the highway could absorb the kinetic energy of high speed cars to recharge other slow cars (in need of energy) on the highway through contact of the wheels with the road. This would reduce drastically the infrastructure cost of gas stations. In other words, it would flexibly adapt according to the external circumstances in order to absorb the traffic without the need of additional expensive infrastructures. Change now the highway into a telecommunication network and the car users into terminals and you will get what is known as MFN. One can immediately see the potential of such networks, able to let information and energy [1] flow in a transparent manner. In some sense, the network texture would change opportunistically.

MFN [2-4] are a disruptive technology targeting very high spectral efficiencies beyond the actual known limits. Indeed, in the cellular wireless arena, engineers frequently stumble on the scalability problem that can be summarized by the following sentence: "As the number of cells in the network increases, interference becomes the bottleneck". As networks become more and more dense, classical techniques based on frequency and space reuse or power control are not be able to cope with interference due the increasing number of terminals. On the contrary, MFN do not consider wireless resources as "a cake to be shared" among users but take benefit of the high number of interacting devices to increase the spectral efficiency frontier. In some sense, more devices represent more opportunities to schedule information which enhances the global throughput. In fact, interference is considered as an opportunity rather than a drawback by exploiting intelligently the degrees of freedom of wireless communications.

Space: MIMO (Multiple Input Multiple Output) Networks coordinate the transmission of various base stations and increase the operating signal to noise ratio (SNR) point of the network. It provides the natural multiplexing and diversity gain of MIMO systems (effective at high SNR) and a linear scaling of the capacity with the number of cooperating base stations. Hence, in theory, the only limiting factor for increasing the spectral efficiency is the number of base stations. The technology, adequate for dense networks, relies on sophisticated tools of dirty paper coding and cooperative Beam-forming.

Frequency/Time: Cognitive networks coordinate the transmission over various bands of frequencies by exploiting the vacant bands in idle periods. It requires antennas able to work in a large range of bandwidth for sensing the different signals in the network. The high density of base stations (by reducing the cell size) enables the use of higher frequencies (for which the path loss increases). 


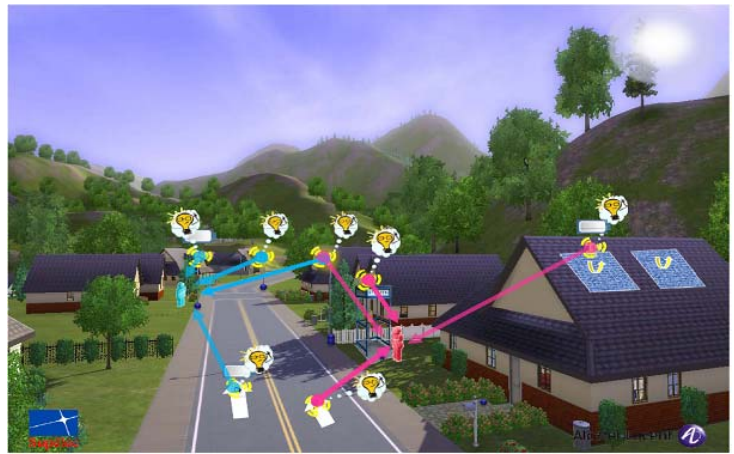

Figure 1. Dense rechargeable base stations which coordinate their transmissions towards users by forming reconfigurable virtual antenna arrays.

User: Opportunistic networks coordinate the transmission to different users in the network, by scheduling intelligently the information to users in good SNR conditions. It turns out that as the number of users increase, the spectral efficiency of opportunistic network increases, as the probability of having a user with a good channel increases with the number of users. These techniques are already deployed in actual systems but will be more advantageous in future highly dense environments.

Mobile Flexible Networks will be at the end dense self-organizing and self-healing secure networks where terminals and base stations interact and self-adapt in an intelligent manner with only a limited amount of regulation (enough to let the terminals/base stations in the network exploit fully the degrees of freedom), being in some sense a bridge between the full centralized and fully decentralized network approaches. The main difference with classical ad-hoc networks [5] (which do not scale appropriately) is that although the terminals may move, one part of the network (base stations) is static or moves ("mini-robot" base stations) at a much lower time scale. This provides means to coordinate the transmission/reception of information and benefit from the various degrees of the spatial gain. The autonomous network made of dense base stations will also be energy harvesting, converting ambient energy into electrical energy, either through wireless recharging [6] or just converting ambient temperature or solar energy into electrical energy. The main idea is to provide a transparent network (from an infrastructure point of view) plug and play type which can be deployed in an instantaneous manner and which can evolve on its own (energy and configuration-wise), without any human intervention. With the multiplicity of standards that are appearing (UMTS, WiFi, WiMAX, LTE), MFN will become more and more necessary. They will have additional features to sense the different technologies, the energy consumption of the terminals and reconfigure (changing from an LTE to UMTS base station if UMTS terminals are present) to adapt to the standards or services to be delivered at a given time.

In the following sections, we will discuss in details the concepts behind MFN as well as the theoretical

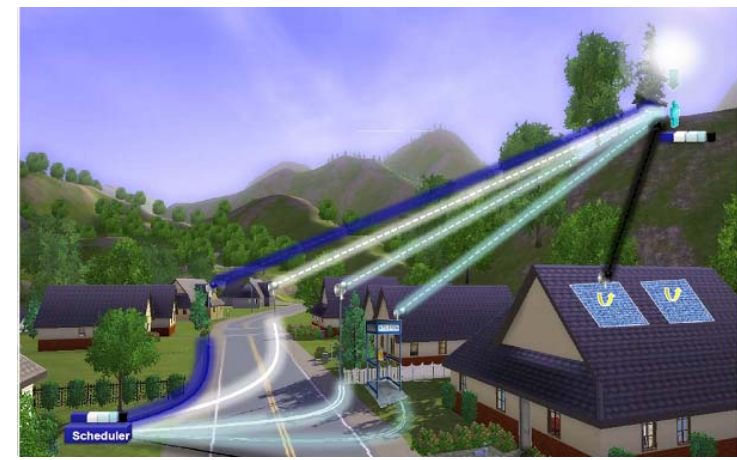

Figure 2. The small reconfigurable base stations are plugged on an heterogeneous infrastructure (powerline, ADSL, fiber optics, ...) to serve the various users in the network. A scheduler splits the packets on the different routes of the wired network.

tools involved in the design of the networks. Section 2 describes in the detailed manner the framework of MFN as well as the new associated spectral efficiency metric. It also provides a historical perspective on the tools required to design MFN. Section 4 provides the challenging topics to be dealt with in order to provide an adequate theory for MFN. Finally, Section 5 is devoted to some conclusions.

\section{Breaking the Spectral Efficiency BARRIER}

\subsection{From $b / s / H z$}

Before introducing the concept of MFN, let us firstly give a brief description of some popular technologies in the domain of wireless communications. In the past twenty years, several generation of standards have been developed going from 2G (known as GSM) to $3 \mathrm{G}$ (UMTS) and now 4G (LTE/WI-MAX, etc.). In each generation, different specific access schemes were applied, such as TDMA (Time Division Multiple Access), CDMA (Code Division Multiple Access) and FDMA/OFDMA (Orthogonal Frequency Division Multiplexing), respectively.

To understand the differences between these access schemes, let us recall one famous example. Suppose that a great number of couples within a room would like to communicate. Each member of the couple would like to talk to his/her own partner and is not interested in what other couples are talking about. In order to make that happen, several possibilities are offered to the couples.

Let us first make an analogy with the case of FDMA (Frequency Division Multiple Access or its advanced version known as OFDM). This system can be represented by walls being built within the room, providing small individual rooms (note that the construction of the walls has a cost). Hence, each couple can go into a small room and are able to communicate without being disturbed by other couples. The differences between FDMA and OFDMA lie in the fact that in the latter case, one can build much thinner walls (thanks to the use of digital Fourier modulator) which optimizes the 
space efficiency.

In the case of TDMA, all the couples would be in the same room. Each couple would talk within a certain period of time, one couple after the other. Hence, a delay would be incurred on their communications depending on the number of couples and the time granted to each of them.

In the case of CDMA, all the couples would be in the same room and would talk simultaneously. However, each couple would speak a different language and would not understand other languages. The code here is the language which is specific to each couple. The language appears here as a filter. For example, a French couple would be able to communicate with each other easily, since they are "sensitive" to their own language and "insensitive" to other foreign languages, because they do not understand the German or the neighboring Chinese couple. In fact, for each couple, foreign languages would be considered as background noise. It is clear that this technique has its own limitation, as one can not add more couples in the room whenever the background noise becomes too "noisy" for any reliable discussion (or that not enough languages are available).

\section{2 ... to $\mathrm{b} / \mathrm{s} / \mathrm{Hz} / \mathrm{m}^{2}$}

Before providing the analogy with couples for MFN, let us go back to the fundamentals of wireless communications. Historically in order to serve the users, service providers have deployed base stations. It immediately appeared that to better serve the users, a dense network of base stations was needed. But at the same time, this would generate harmful interference. In order to alleviate this problem and reduce interference, virtual walls (the different frequency bands bought for each technology) were built (with a non-negligible cost). Unfortunately, "interference" is a misleading word especially when it is not natural but generated by the network. However, MFN consider, on the contrary, interference as a useful signal that can be exploited and build bridges (instead of walls) between the systems. Hence, more interference means more virtual cables that one can use to transmit information towards users. The "highways" are in fact created by the users which are present in the system. In theory, these networks have no capacity limit beside the space constraint but require intelligent devices to acquire the knowledge on the topology at each instant (known as Channel State Information at the transmitter and receiver). The shift from $\mathrm{b} / \mathrm{s} / \mathrm{Hz}$ to $\mathrm{b} / \mathrm{s} / \mathrm{Hz} / \mathrm{m}^{2}$ in terms of spectral efficiency is instrumental in the definition of these networks. This provides a unique opportunity to trade spectrum for space and break the spectral efficiency barrier.

As far as our previous example is concerned, if the people are smart enough to understand/learn all the languages at the same time, the discussion of the neighbor is not considered as noise anymore but potentially useful information that all the couples can jointly help to reach its destination through adequate hops. No shouting is needed anymore and all the couples

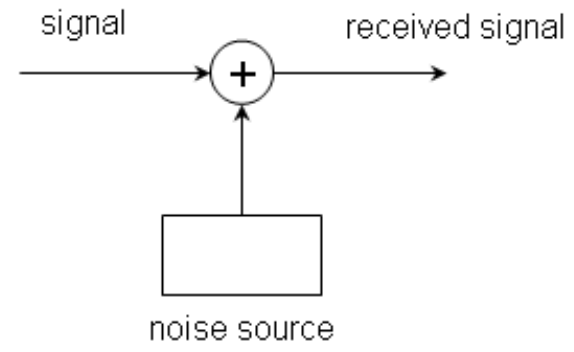

Figure 3. Shannon's approach.

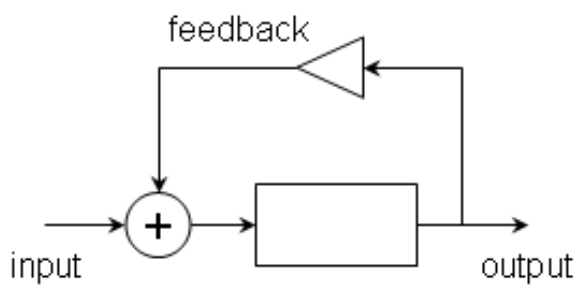

Figure 4. Wiener's approach.

can discuss simultaneously. In fact, more couples in the room mean more opportunities for information to transit.

\section{A Historical Perspective}

\subsection{The Shannon-Wiener Legacy: from 1948 to 2008}

In 1948, two important landmark papers were published. The first one [7] was published by Shannon and introduced a proof of the capacity of a channel with noise. Quite remarkably, Shannon provided a model (which is still of important use today) based on a statistical nature of the communication medium (see Figure 3). Hence, for a given probabilistic model of the medium, he provided the means to compute the exact transmission rate of the information in the system. At the same time, Wiener [8] derived the same capacity formula (without an explicit proof) and introduced the necessary notion of feedback in the communication scheme. Hence, quite remarkably, without the need of an explicit model of the "black box" (see Figure 4), one could theoretically control the output (which is determined by a specific target) based on the feedback mechanism, which provided a measurement of the error induced. The framework and the introduction of feedback is of great interest today in wireless communications, where one has only partial knowledge of the wireless medium. Control theory turns out to be a very neat way of designing the feedback (how many bits of feedback needed, analog or digital, ...).

For the single input single output framework, these two papers were instrumental. Sixty years later, the MIMO Mobile Flexible Network framework in the realm of the cybernetic work of Wiener is much more general (see Figure 5) in the sense that the "black box" has multiple inputs and multiple outputs. The 


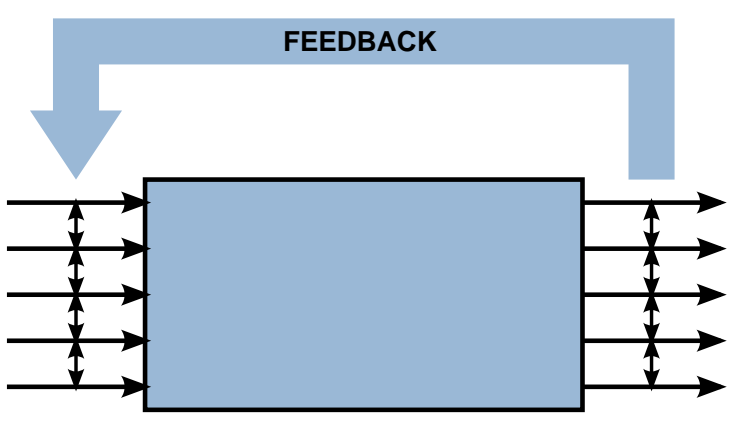

Figure 5. MIMO mobile flexible networks.

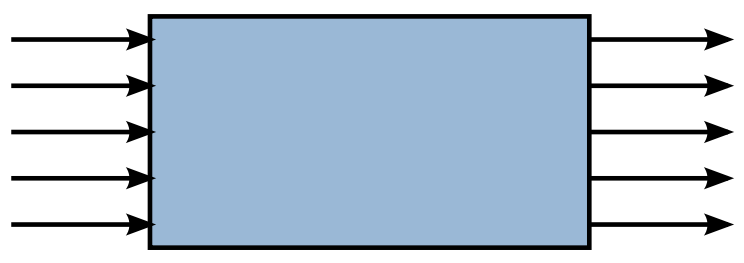

Figure 6. Mutual information approach.

inputs are not necessarily connected or can be partially connected (input 1 can be connected to input 4 for example). The same holds for the outputs (the single user multi-antenna case [9] corresponds to the case where all the inputs and outputs are connected). Moreover, there is a lot of flexibility in the feedback mechanism (typically, output 3 can be only connected to input 1 for example). Finally and this is a major difference with previous works, the designer must learn and control the " black box"

- within a fraction of time

- with finite energy.

This constraint due to the mobility is at the moment extremely hard to cope with as the number of inputs/outputs (the dimensionality of the system) is of the same order as the time scale (in terms of number of time symbols) changes of the box.

\subsection{Information Transfer for Flexible Networks}

Let us suppose that all the inputs are related to the outputs in a linear form with additive noise:

$$
\mathbf{y}=\mathbf{W x}+\mathbf{n}
$$

W represents here the "black box". Moreover, we suppose no mobility ( $\mathbf{W}$ stays constant) and all in the inputs are connected. The same holds for the outputs. Let us compute now the information transfer in the classical sense (infinite delay, Gaussian inputs, Gaussian noise, channel perfectly known at the receiver, ...).

In Shannon's point of view, one needs to provide a probabilistic model of Figure 6. In this case, the capacity is given by ${ }^{1}$ :

\footnotetext{
${ }^{1}$ The differential entropy of a complex Gaussian vector $\mathbf{x}$ with covariance $\mathbf{Q}$ is given by $\log _{2} \operatorname{det}(\pi e \mathbf{Q})$ [10].
}

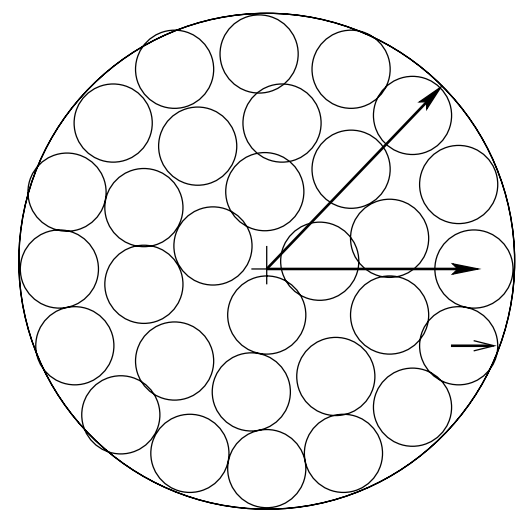

Figure 7. Sphere packing approach.

$$
\begin{aligned}
C & =H(\mathbf{y})-H(\mathbf{y} \mid \mathbf{x}) \\
& =\log \operatorname{det}\left(\pi e \mathbf{R}_{y}\right)-\log \operatorname{det}\left(\pi e \mathbf{R}_{n}\right) \\
& =\log \left(\frac{\operatorname{det}\left(\mathbf{R}_{y}\right)}{\operatorname{det}\left(\mathbf{R}_{n}\right)}\right)
\end{aligned}
$$

In Wiener's point of view (see Figure 7), the vector output $\mathbf{y}$ lies in a small cell centered around the vector $\mathbf{W x}$. The error is due to the noise $\mathbf{n}$. The average volume of the noise cell is proportional ( $\mathbf{n}$ is random multidimensional vector) to $\operatorname{det}\left(\mathbf{R}_{n}\right)$. The average volume of the received signal cell is proportional to $\operatorname{det}\left(\mathbf{R}_{y}\right)$. The number of small cells that one can pack in the big sphere (number of distinguishable vectors $\mathbf{W x}$ ) is given by the ratio:

$$
M=\frac{\operatorname{det}\left(\mathbf{R}_{y}\right)}{\operatorname{det}\left(\mathbf{R}_{n}\right)}
$$

In general, one has an estimate $\hat{\mathbf{y}}$ of $\mathbf{y}$. However, the analysis is the same and one can show that $M$ is maximized (the capacity is achieved) when one has a minimum error $\left(\operatorname{det}\left(\mathbf{R}_{n}\right)\right)$. The goal of the feedback mechanism, through the process of control, will exactly try to minimize the error and maximize $M$.

When the mean square error is minimum, one can communicate reliably using a codebook of size $M$, which contains $\log _{2}(M)$ information bits.

In the classical sense, the eigenvalues of

- the covariance $\mathbf{R}_{y}$ of the output signal

- the covariance $\mathbf{R}_{n}$ of the error

fully describe the information transfer in the system. Unfortunately, as we will see later on, in the case of Mobile Flexible Networks, a thinking of a new sort needs to be developed to determine the information transfer rate.

\section{Research Themes for the Development of Mobile Flexible Networks}

In his early papers, Shannon [11, 12] already described the first learning devices and discussed theoretical developments of self-reproducing machines in very sim- 
plistic cases. Nowadays, MFN's face much broader and complex problems due to three facts:

- the systems are heterogeneous in transmit power, frequencies, range, QoS requirements, spectral efficiency and standards.

- there may be limited or no communication between different systems and decisions have to be made based on such distributed information.

- the systems change rapidly and the flexible network needs to adapt fast and anticipate future evolutions.

One of the most challenging problems in the development of MFN's is to manage complexity and develop the right tools to reason about the spatial and temporal dynamics of complex systems. In order to break the spectrum efficiency barrier, the research should be inter-disciplinary and is a blend of:

1) Maximum entropy methods [13] to build devices which can carry plausible reasoning.

2) Game theoretic techniques [14] (based on rational players) to promote distributed resource allocation schemes.

3) Random matrix [15] to reduce the dimensionality of the network i.e. find the parameters of interest in a network rather than optimizing through simulations with " 1 billion" parameters.

4) Free probability theory [16] to provide tools to infer on the statistics of the network within a finite window of observation.

5) Control theory [8] to understand the use of feedback/signaling mechanisms.

6) Physicsling mechanisms.

7) Physics [17] to study how energy can be processed, stored, and transferred in the network.

8) Network information theory [18] to understand the fundamental network limits achievable with intelligent devices.

9) Wireless cryptography [19] to understand the rate reduction of secure systems in highly mobile environments.

10) Probability theory and Statistical signal processing [20], which provides efficient methods to estimate the high number of parameters involved in uncertain MFN's topologies.

11) Evolutionary biology [21] to understand the dynamics of the system, in particular the changes and convergence towards equilibria over time.

12) Discrete mathematics [22] for constructing distributed space time codes which are delay tolerant.

In the following sections, we discuss the challenges and research opportunities in the field of MFN's.

\subsection{The Uncertainty Foundations}

MFN's take benefit of the high number of interacting devices to schedule adequately information. However, as the number of devices increases, the number of degrees of freedom to estimate in the network increases as well, which incurs a diminishing return on the effective capacity scaling of these networks. In the case of finite

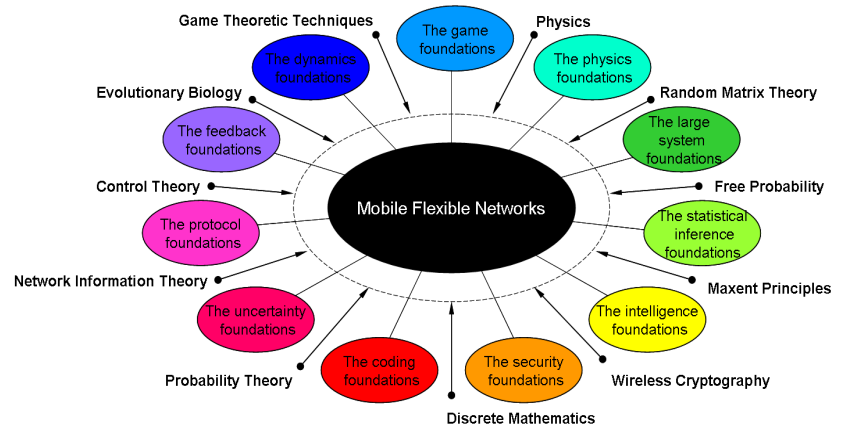

Figure 8. The theoretical foundations of MFN's.

energy devices, there is an actual limit on the number of degrees of freedom that one has to take into account (typically one should only coordinate a subset of the base stations on a subset number of carriers which depends on the time/frequency/space coherence of the network topology). This is reminiscent of previous results already obtained in the analysis of the capacity of non-coherent multiple antenna systems [23] ,where it is shown that one should use only a subset of the transmitting antennas in MIMO systems related to the coherence time. In the frequency domain, similar results [24] have also shown that for finite energy devices, one should only use a subset of the bandwidth, related to the coherence bandwidth. Indeed, as the energy is finite, the transmitter will spread its channel estimation energy across all the degrees of freedom incurring an inadequate estimation of the different degrees at the receiver. Therefore, the receiver reduces considerably its ability to recover the data as the number of degrees increases, compared to the case where only a subset is selected. One main research topic should be to extend the previous results to MFN's where users/base stations/mobility pattern/bandwidth play all an identical role and provide an expression of the non-coherent capacity of these systems for design purposes.

\subsection{The Large Dimension Foundations}

In the design and analysis of wireless networks, researchers frequently stumble on the scalability problem. In other words, as the number of nodes in the network increases, problems become harder to solve. See the following examples:

- In routing: As the network size increases, routes consist of an increasing number of nodes, and so they are increasingly susceptible to node mobility and channel fading.

- In transmission scheduling: The determination of the maximum number of non-conflicting transmissions in a graph is a NP-complete problem.

- In capacity of wireless networks: As the number of nodes increases, the determination of the precise capacity region becomes an intractable problem.

Nevertheless when the system is sufficiently large, one may hope that a macroscopic view would provide a more useful abstraction of the network. The properties of the new macroscopic model would, however, 
consider microscopic considerations. Indeed we may sacrifice some details, but this macroscopic view will preserve sufficient information to allow a meaningful network optimization solution and the derivation of insightful results in a wide range of settings.

There has been some recent works showing how physics tools and random matrix theory [25] can capture most of the complexity of random networks in order to obtain some insightful features on the ensemble behavior. Starting from the work by P. Jacquet [26] in that area, a number of research groups have worked on massively dense ad-hoc networks using tools from geometrical optics [26], percolation theory [27], continuum models [28, 29] as well as electrostatics [30, 31]. The challenges ahead should be to pursue this analogy with more sophisticated models related to physics [32].

\subsection{The Statistical Inference Foundations}

A question that naturally arises in mobile networks is the following: "From a set of $K$ noisy and non necessarily equally sampled measurements, what can a terminal extract in terms of useful information on the network? Moreover, once this information has been extracted, how can the terminal exploit (through dissemination, decision process, etc.) that information?". The problem of MFN's is that, in general, the coherence time (in the number of time symbols) of the network is as the same order as the number of devices in the network. Hence, classical signal processing tools (to calculate statistics such as the covariance from which one extracts information in the case of Gaussian signals) which are based on asymptotics cannot cope anymore with the limited time opportunity which is left to understand the network. Recent results on free deconvolution [33] have been quite successfully applied in recent works [34] to extract information (where the information was related solely to the eigenvalues of the random network) for very simple models, i.e. the case where network is unitarily invariant (meaning that basically, some kind of invariance or symmetry in the problem). Extensions to more sophisticated models need to be addressed in the case of MFN's as in $[35,36]$.

\subsection{The Security Foundations}

Security is a main issue in MFN's where perfect secure transmissions become increasingly difficult to obtain in highly mobile environments. Security is, in general, dealt at the protocol layer through the use of key exchanges [37], which is very adapted to a highly reliable physical layer and centralized network. However, in the case of wireless block fading channels (where capacity, in the sense of no error in the transmission, can not be achieved) and decentralized networks, cryptographic schemes are not adapted anymore. Moreover, in the case of mobility, decentralized secure protocols incur a huge hit on the useful rate. The question is not "how many bits one can transfer in the network without errors" but "how many secure bits one can transfer in the network without error". The gap between capacity and secrecy capacity may not be negligible at all and recent results in the physical layer security community sustain this claim. In his seminal work [19], Shannon formalized the concepts of capacity (as a transmission efficiency measure) and equivocation (as a measure of secrecy). While the concept of capacity has been extended to fading channels with the introduction of concepts like the outage capacity or the ergodic capacity, similar paths are yet to be developed concerning equivocation. Basically, for secure MFN's, multiuser secrecy concepts for fading channels [38] should be better formalized to understand the rate reduction due to secrecy. Moreover, secrecy code design [39] based on secrecy concepts are still a matter of research and should be the next challenge for capacity code designers. Another research direction is based on channel reciprocity concepts for which decentralized devices can construct keys based on common shared resource (channel reciprocity, temperature, etc.) [40].

\subsection{The Protocol Foundations}

One of the great issues in the design of MFN's is to propose a general theory upon information theory where the constraints of delay/protocol overhead are taken into account in the notion of capacity. The general network information theory research takes its roots with the work of Gallager in 1973 [18] who offered the pioneering vision for wired networks. In the wireless field, the issue is all the more important that the classical layering approach of communications is not adequate. Although many papers deal with the now famed cross layer design, the issues addressed are still specific and tailored to a given technology which rip off the gains provided by the reusability of the different protocol stacks in the famous seven layer Open System Interconnection (OSI) framework (with the clear distinction between the physical, the link and higher layers). Hence, what is gained in terms of rates is lost by the lack of its general applicability and therefore requires a full new design of a protocol for each new technology. This is not so appealing for MFN's, which build on the contrary bridges between systems. We still need to provide cross-layer designs for more general classes of communications schemes (typically for slow to highly mobile networks with a smooth transition between the two).

\subsection{The Dynamics Foundations}

In many aspects, the design of networks has been made at the equilibria state, i.e. engineers optimize the parameters when the network has reached its equilibria. The great majority of results focus on the efficiency, the type (Nash if game theoretical tools are used) and performance of the equilibria state. However, very few look at the dynamics to reach the equilibria. This problem becomes all the more important as the number of iterations to reach the equilibria is a critical issue in MFN's, since its network topology might be changed already before the network converges to the equilibria state. The channel state of knowledge window or the stationary time of the environment needs to be at least 
greater than the convergence time of the algorithms. Due to the very complex nature of the problem, new tools and a thinking of new sort need to be developed taking into account evolution and learning aspects [4143]. Moreover, instead of looking at how equilibria (which in any case will happen only for a short period of time) can be maintained, one should better focus on tools to understand how new equilibria states emerge from non-equilibrium situations.

\subsection{The Feedback Foundations}

In his seminal work [8], Wiener introduced the notion of feedback where the channel was seen as a black box and the target could be controlled through the design of the input signal with respect to the feedback signal. Basically, instead of trying to understand specifically the complex nature of the black box (which changes also due to mobility), the genuine idea of Wiener was to show that one needs only information on the input and output of the channel (through adequate feedback) to fully control the targeted aim. Quite remarkably, this formalism is still up to date in MFN's. The channel state information at the various transmitters can only be acquired through a smart feedback process, which should be combined with the transmitted signal. It turns out that in many cases, one can completely control the target quality of service by learning the output of the channel box, without going into the specifics of the modeling. This is a very promising way of looking at the problem and provides important simplifications in the problem design. This output can then be fed back to the transmitters partially (to reduce overhead) or completely in an analog or digital way.

\subsection{The Game Foundations}

Classical cellular networks have been up to now centralized structures where the base station controls the different wireless devices. It turns out that due to the different nature of the devices (based on different technologies with different consumption criteria) as well as the high mobility of the network, MFN's are going towards more decentralized resource allocations schemes where intelligence is provided at each device. This provides a natural framework for new tools like game theory to understand and design network where devices are in competition and cooperation. The devices can form small virtual networks (coalitions games) [44] or coordinate their strategies (correlated games) [45] depending on the type and amount of information at hand (Bayesian games) [46]. The goal is to reduce the overhead of protocol design of central structures, which are enable to cope with high mobility scenarios. Quite remarkably, work still needs to be done in order to find the optimal split of intelligence between the network and the devices. This is very specific to the mobility pattern. Indeed, for low mobility or static topologies, all the information can be centralized in one structure and all the processing burden can be made by the fixed network. For high mobility scenarios, where information dissemination can have an important overhead cost, it is more suitable to have devices which take decisions on their own.

\subsection{The Physics Foundations}

MFN's intend to design communication schemes that take into account the battery state of mobile devices. Typically, the network would switch from one technology to another depending on the energy consumption. Moreover, neighboring autonomous devices within a given very close range could also transfer wireless energy between them to recharge batteries. It is quite remarkable that the pioneering work of Tesla [1] has only recently led to wireless non-radiative energy transfer [6] research (based on electromagnetic resonance). The mobility of the network brings here again the possibility of considering energy as a transferable utility. Therefore, the whole problem of the design of MFN's could be thought in the following terms, using the analogy between information and energy [17, 47]: "For a given total energy of the system, how many bits can be stored/transfered in the wireless network?". The ultimate capacity design is then nothing else than a reinterpretation of Brillouin's work [48] on the cost of acquiring information.

\subsection{The Intelligence Foundations}

MFN's will be mostly decentralized structures where devices will take decisions on their own. In this respect, it is important to develop methodologies such as two autonomous devices with the same state of information on the network take the same decision (or develop the same models and algorithms). This is at the heart of what are known as consistency axioms [49], which enable to build devices behaving according to certain desiderata. In the specific case where the desiderata are consistency axioms of the following nature:

- Uniqueness: If the device solves the same problem twice the same way then the same answer should result both times.

- Invariance: If the device solves the same problem in two different coordinate systems then the same answer should result both times.

- System independence: It should not matter whether the device accounts for independent information about independent systems separately in terms of different densities or together in terms of a joint density.

- Subset independence: It should not matter whether the device treats an independent subset of system states in terms of a separate conditional density or in terms of the full system density.

Shore et al. [50] proved that the principle of maximum entropy [13] is the correct method of inference for the device when given new information in terms of expected values. They proved that maximizing entropy is correct in the following sense: maximizing any function but entropy will lead to inconsistencies unless that function and entropy have the same maximum. The case of information which is not given in terms of 
expected values is still a matter of research and has been partially touched upon in [25]. Moreover, although the maximum entropy principle provides a method to encode information into a number (between 0 and 1 called plausibility), it would be interesting to pursue the extension to a vector encoding process where not only the plausibility of a statement is considered but other features relevant to intelligent autonomous devices.

\subsection{The Coding Foundations}

The results of coding theory are mostly related to centralized equilibrium information theory where infinite delay is available as well as the ability to control all aspects of the entries of the system (as in single user multi-antenna systems [9]). After more than sixty years of research, the shift from theory to practice has been finally realized with the advent of turbo-codes [51] and LDPC codes [52]. However, if one takes into account the context of Mobile Flexible Networks where mobility and distributed resource allocation schemes are required, classical coding techniques turn out to be useless (due to the delays constraints). As far as distributed coding results are concerned, the recent work in [53] paves the path of un-synchronized base stations with distributed robust space-time codes. Taking into account the features of bursty transmission with delay requirements is also an important topic in coding theory and has only recently found a very nice framework in [54]. Extensions of this work to the highly unreliable channel (with channel estimation, frequency selective channel, ...) nature of Mobile Flexible Networks should be considered.

\section{Conclusion}

In this paper, we have discussed the challenges facing the design of MFN's. These networks, if adequately designed, could solve the shortage of spectrum problem by trading spectrum for space. This comes through the use of intelligence and cognition which permits each terminal to acquire knowledge on the topology of the network. This knowledge provides the terminals means to schedule information adequately and benefit from the high number of virtual links (deployed by the high number of interacting devices). The theoretical foundations of these networks are still a matter of research and should be the next challenge in the wireless arena.

\section{ACKNOWLEDGMENT}

This work was supported by Alcatel-Lucent within the Alcatel-Lucent chair on Flexible Radio.

\section{REFERENCES}

[1] N. Tesla, "The transmission of electrical energy without wires," Electrical World, March 51904.

[2] S. Haykin, "Cognitive radio: Brain-empowered wireless communications," IEEE Journal on Selected Areas in Communications, vol. 23, no. 2, pp. 201-220, 2005.
[3] R. Hachemani, J. Palicot, and C. Moy, "The "sensorial radio bubble" for cognitive radio terminals," in The XXIX General Assembly of the International Union of Radio Science (URSI), Chicago (USA), August 2008.

[4] J. Mitola III, "Cognitive radio: An integrated agent architecture for software defined radio," Ph.D. dissertation, Royal Institute of Technology (KTH) Stockholm, 2000.

[5] P. Gupta and P. R. Kumar, "The capacity of wireless networks," IEEE Transactions on Information Theory, vol. 46, no. 2, pp. 388-404, 2000.

[6] A. Karalis, J. Joannopoulos, and M. Soljacic, "Efficient wireless non-radiative mid-range energy transfer," $A n$ nals of Physics, vol. 323, no. 1, pp. 34-48, 2008.

[7] C. E. Shannon, "A mathematical theory of communication," The Bell Labs Technical Journal, vol. 27, pp. 379-457, 623-656, July-October 1948.

[8] N. Wiener, Cybernetics. New York: J. Wiley and Sons, Inc., 1948.

[9] E. Telatar, "Capacity of multi-antenna gaussian channels," European Transactions on Telecommunications, vol. 10, no. 6, pp. 585-596, Nov. 1999.

[10] T. Cover and J. Thomas, Elements of Information Theory. Wiley, 1991.

[11] C. Shannon, "Programming a computer for playing chess," Philosophical Magazine, vol. 41, pp. 256-275, March 1950.

[12] _ " "Computers and automata," Proceedings of the IRE, vol. 41, pp. 1234-1241, October 1953.

[13] E. T. Jaynes, Probability Theory: The Logic of Science. Cambridge, 2003.

[14] J. von Neumann and O. Morgenstern, Theory of Games and Economic Behavior. Princeton, NJ: Princeton University Press, 1944.

[15] V. Marchenko and L. Pastur, "Distribution of eigenvalues for some sets of random matrices," Math USSR-Sbornik, vol. 1, no. 4, pp. 457-483, 1967.

[16] D. Voiculescu, "Multiplication of certain non-commuting random variables," Journal of Operator Theory, vol. 18, pp. 223-235, 1987.

[17] C. H. Bennett and R. Landauer, "The fundamental physical limits of computation," Scientific American, vol. 253, no. 1, pp. 48-56, July 1985.

[18] R. Gallager, "Basic limits on protocol information in data communication networks," IEEE Transactions on Information Theory, vol. 22, no. 4, pp. 385-398, 1976.

[19] C. E. Shannon, "Communication theory of secrecy systems," The Bell Labs Technical Journal, vol. 28, no. 4, pp. 656-715, May 1949.

[20] A. V. Oppenheim, Digital Signal Processing. Englewood Cliffs, NJ: Prentice-Hall, 1975.

[21] J. M. Smith, Evolution and the Theory of Games. Cambridge: Cambridge University Press, 1982.

[22] G. Verriest and E. Picard, OEuvres mathématiques d'Évariste Galois publiées en 1897. Paris: Gauthier-Villars, 1951.

[23] L. Zheng and D. N. C. Tse, "Communication on the Grassmann manifold: A geometric approach to the noncoherent multiple-antenna channel," IEEE Transactions on Information Theory, vol. 48, no. 2, pp. 359-383, 2002.

[24] M. Borgmann and H. Bolcskei, "On the capacity of noncoherent wideband MIMO-OFDM systems," in Proc. IEEE Int. Symposium on Information Theory (ISIT), 2005, pp. 651-655.

[25] M. Debbah and R. Muller, "MIMO channel modelling and the principle of maximum entropy," IEEE Transactions on Information Theory, vol. 51, no. 5, pp. 1667-1690, 2005.

[26] P. Jacquet, "Geometry of information propagation in massively dense ad hoc networks," in Proc. 5th ACM Int. Symposium on Mobile Ad Hoc Networking and Computing (MobiHoc), New York, NY, USA, 2004, pp. 157-162.

[27] M. Franceschetti, O. Dousse, D. Tse, and P. Tiran, "Clos- 
ing the gap in the capacity of random wireless networks," in Proc. IEEE Int. Symposium on Information Theory (ISIT), 2004.

[28] M. Beckmann, "A continuum model of transportation," Econometrica, vol. 20, pp. 643-660, 1952.

[29] P. Daniele and A. Maugeri, "Variational inequalities and discrete and continuum models of network equilibrium problems," Mathematical and Computer Modelling, vol. 35, no. 5-6, pp. 689-708, 2002.

[30] S. Toumpis, "Optimal design and operation of massively dense wireless networks: or how to solve 21st century problems using 19th century mathematics," in Proc. Workshop on Interdisciplinary Systems Approach in Performance Evaluation and Design of Computer $\mathcal{E}$ Communication Systems, New York, NY, USA, 2006.

[31] S. Toumpis and L. Tassiulas, "Optimal deployment of large wireless sensor networks," IEEE Transactions on Information Theory, vol. 52, no. 7, pp. 2935-2953, 2006.

[32] E. Altman, A. Silva, P. Bernhard, and M. Debbah, "Continuum equilibria for routing in dense ad-hoc networks," in Proc. 45th Annual Allerton Conference on Communication, Control, and Computing, Illinois, USA, September 26-28 2007.

[33] O. Ryan and M. Debbah, "Free deconvolution for signal processing applications," IEEE Transactions on Information Theory, 2008, second round review. [Online]. Available: http://arxiv.org/abs/cs.IT/0701025

[34] —, "Channel capacity estimation using freeprobability theory," IEEE Transactions on Signal Processing, vol. 56, no. 11, pp. 5654-5667, 2008.

[35] _-, "Random Vandermonde matrices-part I: Fundamental results," IEEE Transactions on Information Theory, 2008, submitted. [Online]. Available: http://arxiv.org/abs/0802.3570v1

[36] - "Random Vandermonde matrices-part II: Applications," IEEE Transactions on Information Theory, 2008, submitted. [Online]. Available: http://arxiv.org/abs/0802.3572v1

[37] W. Diffie and M. Hellman, "New directions in cryptography," IEEE Transactions on Information Theory, vol. 22, no. 6, pp. 644-654, 1976.

[38] Y. Liang, H. V. Poor, and S. Shamai, "Secure communication over fading channels," IEEE Transactions on Information Theory, vol. 54, no. 6, pp. 2470-2492, 2008.

[39] R. Liu, Y. Liang, H. V. Poor, and P. Spasojevic, "Secure nested codes for type II wiretap channels," in Proc. IEEE Information Theory Workshop (ITW), 2007, pp. 337-342.

[40] R. Ahlswede and I. Csiszar, "Common randomness in information theory and cryptography. I. Secret sharing," IEEE Transactions on Information Theory, vol. 39, no. 4, pp. 1121-1132, 1993.

[41] W. B. Arthur, "Inductive reasoning and bounded rationality (the el farol problem)," The American Economic Review (Papers and Proceedings of the Hundred and Sixth Annual Meeting of the American Economic Association), vol. 84, pp. 406-411, May 1994.

[42] A. Rustichini, "Optimal properties of stimulus-response learning models," Games and Economic Behavior, vol. 29, no. $1-2$, pp. $244-273,1999$.

[43] P. Mertikopoulos and A. Moustakas, "Correlated anarchy in overlapping wireless networks," IEEE Journal on Selected Areas in Communications, vol. 26, no. 7, pp. 1160$1169,2008$.

[44] W. Saad, Z. Han, M. Debbah, and A. Hjorungnes, “Network formation games for distributed uplink tree construction in IEEE 802.16J networks," in Proc. IEEE Global Telecommunications Conference (GLOBECOM), 2008, pp. 15.

[45] R. Aumann, "Subjectivity and correlation in randomized strategies," Journal of Mathematical Economics, vol. 1, pp. 67-96, 1974.

[46] D. Fudenberg and J. Tirole, Game Theory. The MIT Press, 1991.
[47] C. H. Bennett, "Demons, engines and the second law," Scientific American, vol. 257, no. 5, pp. 108-116, Nov 1987.

[48] L. Brillouin, Science and Information Theory, 2nd ed. New York: Academic Press, 1962.

[49] R. T. Cox, "Probability, frequency and reasonable expectation," American Journal of Physics, vol. 14, no. 1, pp. $1-13,1946$.

[50] J. Shore and R. Johnson, "Axiomatic derivation of the principle of maximum entropy and the principle of minimum cross-entropy," IEEE Transactions on Information Theory, vol. 26, no. 1, pp. 26-37, 1980.

[51] C. Berrou, A. Glavieux, and P. Thitimajshima, "Near Shannon limit error-correcting coding and decoding: Turbo-codes. 1," in Proc. IEEE Int. Conference on Communications (ICC), vol. 2, Geneva, Switzerland, May 1993 pp. 1064-1070.

[52] R. G. Gallager, Low Density Parity Check Codes. Cambridge, MA, USA: MIT Press, 1963.

[53] M. O. Damen and A. R. Hammons, "Delay-tolerant distributed-TAST codes for cooperative diversity," IEEE Transactions on Information Theory, vol. 53, no. 10, pp. 3755-3773, 2007.

[54] Y. Polyanskiy, H. V. Poor, and S. Verdu, "Channel coding rate in the finite blocklength regime," IEEE Transactions on Information Theory, vol. 56, no. 5, pp. 2307-2359, 2010.

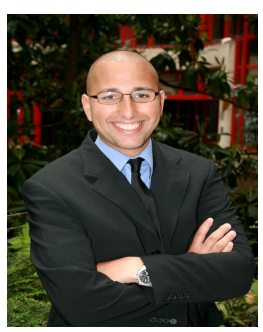

Mérouane Debbah was born in Madrid, Spain. He entered the Ecole Normale Suprieure de Cachan (France) in 1996 where he received his M.Sc and Ph.D. degrees in 1999 and 2002, respectively. From 1999 to 2002, he worked for Motorola Labs on Wireless Local Area Networks and prospective fourth generation systems. From 2002 until 2003, he was appointed Senior Researcher at the Vienna Research Center for Telecommunications (FTW) (Vienna, Austria) working on MIMO wireless channel modeling issues. From 2003 until 2007, he joined the Mobile Communications department of the Institut Eurecom (Sophia Antipolis, France) as an Assistant Professor. He is presently a Professor at Supelec (Gif-sur-Yvette, France), holder of the Alcatel-Lucent Chair on Flexible Radio. His research interests are in information theory, signal processing and wireless communications. Mérouane Debbah is the recipient of the "Mario Boella" prize award in 2005, the 2007 General Symposium IEEE GLOBECOM best paper award, the WiOpt 2009 best paper award, the $\mathrm{N}++$ best paper award as well as the Valuetools 2007, Valuetools 2008 and CrownCom 2009 best student paper awards. He is a WWRF fellow. 